\title{
Sjögren Syndrome in Systemic Lupus Erythematosus: A Subset Characterized by a Systemic Inflammatory State
}

\author{
Guillermo Ruacho, Marika Kvarnström, Agneta Zickert, Vilija Oke, Johan Rönnelid, \\ Susanna Eketjäll, Kerstin Elvin, Iva Gunnarsson, and Elisabet Svenungsson (i)
}

\begin{abstract}
Objective. An often-neglected subset of patients with systemic lupus erythematosus (SLE) is those with secondary Sjögren syndrome (SLE-sSS). Further, primary SS overlaps and can be difficult to delineate from SLE. To shed light on the SLE-sSS subset, we investigated a large and well-characterized SLE cohort, comparing patients with SLE-sSS and SLE patients without SS (SLE-nonsSS) and controls.

Methods. We included 504 consecutive patients with SLE, fulfilling the 1982 revised American College of Rheumatology criteria, and 319 controls from the general population, matched for age and sex to the first 319 patients. SLE-sSS was defined according to the American-European Consensus Criteria (AECC). A thorough clinical examination, including subjective and objective quantifications of sicca symptoms, was performed in all participants. Autoantibodies and 20 selected cytokines were measured by luminex and multiplex analysis, respectively.

Results. SLE-sSS, as defined by AECC, occurred in 23\% of the patients with SLE. In comparison to SLE-nonsSS, the SLE-sSS group was older and more frequently female. Leukopenia and peripheral neuropathy were more frequent and nephritis less frequent. Circulating levels of 6/20 investigated proinflammatory cytokines [tumor necrosis factor- $\alpha$, interleukin (IL) 6 , monocyte chemoattractant protein 4 , macrophage inflammatory protein $1 \beta$, IL-12/IL-23p40, and interferon $\gamma$-induced protein 10], total IgG, anti-SSA/Ro52, anti-SSA/Ro60, anti-SSB/La antibodies, and rheumatoid factor (IgM and $\operatorname{IgA}$ ) were higher in the SLE-sSS group ( $\mathrm{p}<0.05$ for all comparisons).

Conclusion. The frequency of SLE-sSS increased with age and affected roughly one-quarter of all patients with SLE. Despite less internal organ involvement, a systemic inflammatory state with high levels of proinflammatory cytokines is present in the SLE-sSS subgroup. This is a novel observation that may affect future understanding and treatment of the SLE-sSS subset. (First Release April 15 2020; J Rheumatol 2020;47:865-75; doi:10.3899/jrheum.190250)
\end{abstract}

\section{Key Indexing Terms:}

SYSTEMIC LUPUS ERYTHEMATOSUS SJÖGREN SYNDROME SICCA SYMPTOMS SJÖGREN SYNDROME ANTIGEN A SJÖGREN SYNDROME ANTIGEN B CYTOKINES

From the Department of Medicine Solna, Division of Rheumatology, Karolinska Institutet, Stockholm; Karolinska University Hospital, Stockholm; Karolinska Institutet/AstraZeneca Integrated Cardio Metabolic Centre (KI-AZ ICMC), Stockholm; Department of Medicine Solna, Division of Immunology and Allergy, Karolinska Institutet, Stockholm; Center for Clinical Research, Uppsala University, Sörmland; Department of Immunology, Genetics and Pathology, Uppsala University, Uppsala; Cardiovascular, Renal and Metabolism, Innovative Medicines and Early Development (IMED) Biotech Unit, AstraZeneca, Huddinge, Sweden.

Supported by The Swedish Research Council (grant no. 201433867), Swedish Heart-Lung Foundation (grant nos. 20130430, 20130552,20170257), Stockholm County Council (ALF, grant nos. 2014016, 20170038), The King Gustaf V 80th Birthday Fund (grant no. FAI-2017-0390), The Swedish Rheumatism Association (grant no. R-739631), Karolinska Institutet's Foundations, The Swedish Society of Medicine, Ingegerd Johansson's foundation (SLS-713911), The Swedish Dental Society, and clinical research grants from Sörmland county (no: DLL-614351, DLL-648991). S. Eketjäll is an employee at AstraZeneca. AstraZeneca provided reagents for the cytokine analyses, but AstraZeneca had no influence on the analyses or on the presentations in this manuscript.

G. Ruacho, DMD, Department of Medicine Solna, Division of
Rheumatology, Karolinska Institutet, and Center for Clinical Research, Uppsala University; M. Kvarnström, MD, PhD, Department of Medicine Solna, Division of Rheumatology, Karolinska Institutet, and Karolinska University Hospital; A. Zickert, $M D, P h D$, Department of Medicine Solna, Division of Rheumatology, Karolinska Institutet, and Karolinska University Hospital; V. Oke, MD, PhD, Department of Medicine Solna, Division of Rheumatology, Karolinska Institutet, and Karolinska University Hospital; J. Rönnelid, MD, PhD, Department of Immunology, Genetics and Pathology, Uppsala University; S. Eketjäll, PhD, Cardiovascular, Renal and Metabolism, IMED Biotech Unit, AstraZeneca, and KI-AZ ICMC; K. Elvin, MD, PhD, Department of Medicine Solna, Division of Immunology and Allergy, Karolinska Institutet and Karolinska University Hospital; I. Gunnarsson, MD, PhD, Department of Medicine Solna, Division of Rheumatology, Karolinska Institutet, and Karolinska University Hospital; E. Svenungsson, MD, PhD, Department of Medicine Solna, Division of Rheumatology, Karolinska Institutet, and Karolinska University Hospital. G. Ruacho and M. Kvarnström contributed equally to this work.

Address correspondence to Dr. E. Svenungsson, Department of Medicine Solna, Division of Rheumatology D2:01, Karolinska Institutet, SE-171 76 Stockholm, Sweden.E-mail: elisabet.svenungsson@ki.se

Full Release Article. For details see Reprints and Permissions at jrheum.org Accepted for publication August 23, 2019.

Personal non-commercial use only. The Journal of Rheumatology Copyright @ 2020 . All rights reserved. 
Systemic lupus erythematosus (SLE) is a chronic autoimmune systemic disease characterized by enhanced autoantibody production and formation of immune complexes. SLE is also a very heterogeneous condition involving many organ systems, and disease activity varies from persistently mild to life-threatening ${ }^{1}$. A state of systemic inflammation, often associated with complement consumption, enhanced activity in the type I interferon system, as well as high levels of proinflammatory cytokines [e.g., tumor necrosis factor- $\alpha$ (TNF- $\alpha$ ), interleukin 6 (IL-6), IL-8, and interferon $\gamma$-induced protein (IP)-10], is common in SLE ${ }^{2,3,4,5}$. While the general prognosis of SLE has improved, mortality rates remain more than 2-fold higher than in the general population, and cardiovascular diseases constitute a growing share of mortality causes $6,7,8$.

It has become increasingly clear that different subsets of the SLE population exist. Already in 1959, Heaton suggested that Sjögren syndrome (SS) is a chronic and relatively benign form of SLE ${ }^{9}$. More recently, several studies have identified autoantibody clusters/immune phenotypes, which vary in clinical symptoms, biomarkers, and prognosis ${ }^{10,11,12,13,14}$. An SLE phenotype characterized by antibodies to SSA and SSB antigens (also referred to as Ro/La) consistently appeared in these studies, but this supposedly milder SLE subset has so far achieved limited scientific attention.

The diagnosis of SS is a clinical entity, based on dryness of eyes and mouth due to destructive inflammation in the exocrine glands, especially tear and salivary glands. SS can exist in isolation, as primary SS (pSS), or with other rheumatic diseases, referred to as secondary SS (sSS). A major difference according to the 2002 Revised American-European Consensus Criteria (AECC) is the classification where the serologic item (SSA/SSB antibodies) is included for pSS, but not for $\mathrm{SSS}^{15}$. In SLE these autoantibodies are common, usually stable over time, and they appear early, even several years before disease onset ${ }^{16,17}$.

The clinical SLE-sSS phenotype has been described as a mild version of SLE with dominance of skin and joint manifestations and with less severe internal organ involvement, and in particular, less nephritis ${ }^{9,18,19}$. Differences and similarities between pSS and SLE with SS (SLE-sSS) have been studied ${ }^{20}$, but to what extent the inflammatory pattern differs between SLE-sSS and SLE patients without SS (SLE-nonsSS) is not known, and this information may be important regarding treatment perspectives.

In our present study, subjective and objective symptoms of sSS, defined according to $\mathrm{AECC}^{15}$, from a large and well-characterized cohort of consecutive SLE patients and matched controls are presented. To our knowledge, no previous study has investigated patients with SLE and matched population controls for both subjective and objective symptoms of SS and associated SSA/SSB autoantibodies. The primary outcomes were occurrence, and clinical and immunological characteristics of the SLE-sSS subgroup. As secondary outcomes, we performed stratified analyses based on anti-SSA/SSB profiles.

\section{MATERIALS AND METHODS}

Patients and controls. Patients with SLE managed at the Department of Rheumatology, Karolinska University Hospital and Danderyd's Hospital (Stockholm) who fulfilled 4 or more items of the American College of Rheumatology (ACR) 1982 revised classification criteria for SLE ${ }^{21}$ were invited to participate during the inclusion period of February 2004 to December 2014.

Population controls were individually matched for sex, age, and region of residence to the first 319 patients with SLE. The remaining 185 patients with SLE did not have matched controls. Matching was performed through use of the national registration number, which includes date of birth and is coded for sex. The only exclusion criteria were a diagnosis of SLE or SS.

Clinical and routine laboratory characterization. A structured protocol, similar for patients and controls, was used. Medical charts were reviewed. A rheumatologist performed a clinical examination and evaluated general health and features of SLE, including all items according to the ACR 1982 classification criteria ${ }^{21}$. SLE disease activity was determined with both Systemic Lupus Activity Measure (SLAM) ${ }^{22}$ and the SLE Disease Activity Index 2000 (SLEDAI-2K) ${ }^{23}$. Permanent organ damage was evaluated with Systemic Lupus International Collaborating Clinics/ACR Damage Index $(\mathrm{SDI})^{24}$.

All participants were evaluated for the individual items of SS according to the $\mathrm{AECC}^{15}$. Subjective ocular and oral symptoms were recorded as defined by the validated questionnaire. Objective measurements were Schirmer's test and whole unstimulated salivary flow (WUSF) over 15 $\min ^{25}$. For controls, these objective measurements were only performed if subjective symptoms were present. Data on fulfillment of each item were collected. The amount of tears and saliva were recorded. If a salivary gland biopsy had been performed earlier, results were retrieved from the patient's records. Patients who had not had a salivary gland biopsy, when this was needed to determine whether they fulfilled the sSS criteria, were regarded as not having sSS. The attending rheumatologist assessed these patients as not having sSS, and hence that a salivary gland biopsy was not necessary.

Laboratory measurements. Fasting blood samples were drawn at inclusion. Laboratory tests were performed at the Swedish Board for Accreditation and Conformity Assessment (SWEDAC; www.swedac.se)-accredited Clinical Chemistry and Immunology Laboratories at the Karolinska University Hospital. Routine laboratory tests and analyses of complement factors and immunoglobulins were performed on fresh samples according to clinical routine.

Antinuclear antibodies were analyzed by indirect immunofluorescence on HEp-2 cells (Immunoconcepts). Antibodies to specific nuclear antigens (dsDNA, SSA/Ro52, SSA/Ro60, SSB/La, Sm, RNP) and phospholipid-related antigens [cardiolipin IgG, IgM, and $\beta_{2}$-glycoprotein I ( $\beta_{2}$-GPI), IgG $\mathrm{IgM}]$ were analyzed by multiplexed bead technology (Luminex) using BioPlex 2200 system (Bio-Rad) according to the specifications of the manufacturer. Patients who were simultaneously positive for SSA/Ro52, SSA/Ro60, and SSB/La are referred to as triple SSA/SSB-positive. The cutoff for anticardiolipin and anti- $\beta_{2}$-GPI fulfills the 99 th percentile of the general population in Stockholm, as described ${ }^{26}$. Lupus anticoagulant (LAC) was determined using a modified Russell's viper venom method (Biopool) using Bioclot LAC.

IgA, IgG, and IgM rheumatoid factor (RF) were measured with a Phadia2500 instrument at Phadia Thermofisher Uppsala. Cutoffs for RF isotypes were determined as $>95 \%$ specificity compared with 100 blood donors for IgA and IgM RF and for 285 population controls for IgG RF.

Mesoscale Discovery (MSD) multiplex analysis of cytokines EDTA-plasma samples were analyzed on the MSD V-PLEX Human Cytokine 30-plex kit (K15054D; MSD) according to the manufacturer's instructions, as previously reported ${ }^{4}$. In short, the plasma samples were thawed at room temperature and diluted twice for proinflammatory and

Personal non-commercial use only. The Journal of Rheumatology Copyright (C) 2020. All rights reserved. 
cytokine analyses, and 4 times for chemokine analysis in sample diluents. The diluted samples were incubated on the MSD plates for $2 \mathrm{~h}$ at room temperature with shaking. Plates were washed and incubated an additional $2 \mathrm{~h}$ with detection antibodies. After washing, $2 \times$ Read buffer $\mathrm{T}$ was added and the plates were analyzed in a Sector Imager 6000. Calibrator and plasma samples were analyzed in duplicates. Using the MSD Workbench software, the responses of the calibrator concentrations were plotted as log signal unit on the vertical (y) axis versus log concentration on the horizontal (x) axis. A weighted 4-parameter logistic fit equation was used for curve fitting and back calculation of plasma sample concentrations.

Statistical analysis. Patient and control characteristics are presented as mean $\pm \mathrm{SD}$, median (interquartile range), or percentages, depending on data type and distribution.

Groups were compared with the Student t test, Wilcoxon rank-sum test, or chi-square tests as appropriate. We used nonparametric tests when log transformation of continuous variables did not give a nearly normal distribution. Adjustment for age was performed by multiple logistic regression.

Calculations were performed using JMP software (SAS Institute). A 2 -sided $\mathrm{p}$ value $<0.05$ was considered statistically significant.

The local Ethics Committee at Karolinska Institutet approved the study (Dnr 03-556, Dnr 2017/1570-32). All study subjects gave written informed consent to participate in the study.

\section{RESULTS}

The 504 patients and 319 matched controls were well matched for age, but the female percentage was slightly lower among the patients $(86.3 \%$ vs $92.2 \% ; \mathrm{p}=0.01)$. The patients with SLE were $33.9 \pm 15.5$ years at diagnosis, and their disease duration was $12.1 \pm 12.3$ years. Basic characteristics are presented in Table 1.

Patients with SLE-sSS versus SLE-nonsSS. SLE-sSS was present in $23.2 \%$ of the patients with SLE. Patients with SLE-sSS were older at inclusion (54.6 \pm 13.6 vs $43.4 \pm 14.7$ yrs; $\mathrm{p}<0.0001)$ and at SLE onset $(40.4 \pm 15.6$ vs $31.9 \pm 14.9$ $y r s ; p<0.0001)$. The percentage of patients with SLE-sSS increased with age, as demonstrated in Figure 1. The frequency of females was higher in the SLE-sSS group compared to SLE-nonsSS (95.7\% vs 83.4\%; $p=0.0007$; Table 1 ).

We performed additional calculations to illustrate potential bias regarding the 132 patients with SLE who were regarded as SLE-nonsSS by their attending rheumatologist, but could have been reclassified after a biopsy. Results are presented in Supplementary Table 1 (available with the online version of this article).

Autoantibodies. Sjögren-associated autoantibodies SSA/ Ro52, SSA/Ro60, and SSB/La were more common in patients with SLE-sSS versus SLE-nonsSS (47.9\% vs $21.8 \%$, $\mathrm{p}<0.0001 ; 59 \%$ vs $35.9 \%, \mathrm{p}<0.0001 ; 37.6 \%$ vs $18 \%$, $\mathrm{p}<0.0001$, respectively), but $39.3 \%$ of the SLE-sSS patients were negative for all 3 antibodies. The frequency of SSA/Ro52 and SSA/Ro60 was highest among young patients aged 21-30 (Figure 2). Further, RF of the IgM (38.6\% vs $19.9 \% ; \mathrm{p}=0.0005)$ and the $\operatorname{IgA}(45.9 \%$ vs $28.0 \% ; \mathrm{p}=0.004)$ isotypes were more frequent in SLE-sSS as compared to SLE-nonsSS. Anti-dsDNA autoantibodies were nonsignificantly less prevalent in SLE-sSS than among SLE-nonsSS patients $(31.3 \%$ vs $41 \%$; $\mathrm{p}=0.06$; Table 1$)$.
SLE manifestations, disease activity, and damage. In the SLE-sSS group, leukopenia and peripheral neuropathy were more common than in the SLE-nonsSS $(57.3 \%$ vs $45.2 \%$, $\mathrm{p}=0.02$; and $15.4 \%$ vs $7.5 \%, \mathrm{p}=0.01$, respectively), while nephritis was less frequent (31.9\% vs $42.6 \%$; $\mathrm{p}=0.03)$.

Organ damage (SDI score $>1$ ), was more frequent in the SLE-sSS group (73\% vs 59.9\%; $\mathrm{p}=0.01$ ), but this difference did not remain after age adjustment. High disease activity, as measured by SLAM (score $>6$ ) was more common in the SLE-sSS group (67.2\% vs $57.1 \%$; $\mathrm{p}=0.05)$, whereas SLEDAI scores were similar in both groups (Table 1).

Cytokines and other biomarkers. Higher levels of total $\mathrm{IgG}$ characterized the SLE-sSS group $(\mathrm{p}=0.009)$. Cytokines were measured in 432 SLE patients and 315 controls. Of 30 investigated cytokines, 20 were reliably detectable and evaluated; of these, 19/20 were higher in SLE than in controls, as previously reported ${ }^{4}$. When comparing the SLE-sSS to the SLE-nonsSS group, $6 / 20$ cytokines (TNF- $\alpha$, IL-6, MCP-4, MIP-1 $\beta$, IL12/IL-23p40, and IP-10) were upregulated in SLE-sSS (Table 1).

Exclusion of the 132 patients, who could have been reclassified after biopsy, yielded similar results except for IL12/IL-23p40 and IP-10, which no longer differed significantly.

SSA/Ro52. SLE-sSS $(\mathrm{p}<0.0001)$, leukopenia $(\mathrm{p}=0.04)$, and lymphopenia $(\mathrm{p}=0.05)$ were more common among patients with SLE who were positive for SSA/Ro52, whereas malar rash $(\mathrm{p}=0.04)$ and seizures $(\mathrm{p}=0.02)$ were less frequent than in patients negative for SSA/Ro52 (Table 2).

SSA/Ro60. SSA/Ro60-positive patients were more often affected by sSS $(\mathrm{p}<0.0001)$, leukopenia $(\mathrm{p}=0.0006)$, and photosensitivity $(\mathrm{p}=0.02)$, and less affected by discoid lesions $(\mathrm{p}=0.02)$, nephritis $(\mathrm{p}=0.05)$, vasculitis $(\mathrm{p}=0.03)$, and peripheral neuropathy ( $\mathrm{p}=0.02$; Table 2$)$.

$S S B / L a$. In the SSB/La-positive patient group, sSS $(\mathrm{p}<0.0001)$, arthritis $(\mathrm{p}=0.002)$, leukopenia $(\mathrm{p}=0.0003)$, and lymphopenia $(\mathrm{p}=0.05)$ were more common, but nephritis ( $\mathrm{p}=0.0005$ ) was less common (Table 2$)$.

Sicca symptoms. In all investigated groups (SLE-sSS, SLE-nonsSS, and controls), subjective symptoms of ocular and/or oral sicca symptoms were less frequent than objective measurements of reduced tear and saliva production. While subjective symptoms clearly differed between SLE-nonsSS and controls in all age spans, objectively measured tear production was similar in the age spans $>40$ years, and differences were small also for WUSF after the age of 50 years (Figure 3 and Figure 4).

Ocular sicca symptoms. In the SSA/Ro52-, SSA/Ro60-, and SSB/La-positive groups, $41.4 \%, 39.3 \%$, and $44.2 \%$, respectively, presented with subjective ocular sicca symptoms. Figures were similar for the triple SSA/SSB-positive patients (44.9\%; Supplementary Figure 1A, available with the online version of this article). 
Table 1. Basic characteristics of patients with SLE, controls, and SLE-sSS versus SLE-nonsSS.

\begin{tabular}{|c|c|c|c|c|c|c|}
\hline Characteristics & SLE & Controls & $\mathrm{p}$ & SLE-sSS & SLE-nonsSS & $\mathrm{p}$ \\
\hline $\mathrm{N}(\%)$ & $504(100)$ & $319(100)$ & & $117(23.2)$ & $387(76.7)$ & \\
\hline \multicolumn{7}{|c|}{ Basic characteristics, mean \pm SD or $\mathrm{n}(\%)$} \\
\hline Age, yrs & $46.0 \pm 15.2$ & $47.4 \pm 14.6$ & 0.20 & $54.6 \pm 13.6$ & $43.4 \pm 14.7$ & $<0.0001$ \\
\hline Age at SLE onset, yrs & $33.9 \pm 15.5$ & ND & ND & $40.4 \pm 15.6$ & $31.9 \pm 14.9$ & $<0.0001$ \\
\hline Disease duration, yrs & $12.1 \pm 12.3$ & ND & ND & $14.2 \pm 12.8$ & $11.5 \pm 12.1$ & 0.16 \\
\hline No. SLE criteria* & $5.7 \pm 1.4$ & ND & ND & $5.7 \pm 1.5$ & $5.7 \pm 1.4$ & 0.40 \\
\hline Sex, female & $435(86.3)$ & $294(92.2)$ & 0.01 & $112(95.7)$ & $323(83.4)$ & 0.0007 \\
\hline Smoking, current & $96(19.0)$ & $47(14.6)$ & 0.22 & $20(17.1)$ & $76(19.6)$ & 0.58 \\
\hline Smoking, ever & $258(51.2)$ & $151(46.9)$ & 0.22 & $62(53)$ & $196(50.6)$ & 0.65 \\
\hline Primary SS & $108(21.6)$ & ND & ND & $25(21.9)$ & $83(21.6)$ & 0.99 \\
\hline \multicolumn{7}{|c|}{ Autoantibody positivity, n (\%) } \\
\hline Anti-dsDNA & $190 / 491(38.7)$ & $5 / 317(1.6)$ & $<0.0001$ & $36 / 115(31.3)$ & $154 / 376(41)$ & 0.06 \\
\hline Anti-SSA-Ro52 & $140 / 501(27.9)$ & $3 / 317(0.9)$ & $<0.0001$ & $56 / 117(47.9)$ & $84 / 384(21.8)$ & $<0.0001$ \\
\hline Anti-SSA-Ro60 & $206 / 499(41.3)$ & $5 / 317(1.6)$ & $<0.0001$ & $69 / 117(59)$ & $137 / 382(35.9)$ & $<0.0001$ \\
\hline Anti-SSB-La & $113 / 501(22.6)$ & $10 / 317(3.1)$ & $<0.0001$ & $44 / 117(37.6)$ & 69/384 (18) & $<0.0001$ \\
\hline Anti-Sm & $94 / 501(18.8)$ & 1/317 (0.3) & $<0.0001$ & $19 / 117(16.2)$ & 75/384 (19.5) & 0.42 \\
\hline Anti-SmRNP & $130 / 497(26.2)$ & 0/317 (0) & $<0.0001$ & $29 / 116(25)$ & $101 / 381(26.5)$ & 0.74 \\
\hline Anti-RNP 68 & $51 / 500(10.2)$ & 0/317 (0) & $<0.0001$ & $11 / 117(9.4)$ & $40 / 383(10.4)$ & 0.74 \\
\hline Anti-RNP A & $129 / 496(26.0)$ & $10 / 317(3.1)$ & $<0.0001$ & $30(25.6)$ & $99 / 379(26.1)$ & 0.91 \\
\hline $\mathrm{aCL}$ IgG & $108 / 423(25.5)$ & 2/318 (0.6) & $<0.0001$ & 24/97 (24.7) & $84 / 326(25.8)$ & 0.83 \\
\hline $\mathrm{aCL}$ IgM & 26/432 (6.0) & $1(0.3)$ & $<0.0001$ & 8/96 (8.3) & $18 / 336(5.4)$ & 0.32 \\
\hline Anti- $\beta_{2-}$ GPI IgG & $110 / 417(26.4)$ & $2(0.6)$ & $<0.0001$ & $24 / 95(25.3)$ & $86 / 322(26.7)$ & 0.77 \\
\hline Anti- $\beta_{2}$ GPI IgM & 33/434 (7.6) & $1(0.3)$ & $<0.0001$ & 8/96 (8.3) & $25 / 338(7.4)$ & 0.82 \\
\hline RF IgG & $52 / 339(15.3)$ & $10 / 261(3.8)$ & $<0.0001$ & $17 / 80(21.2)$ & $35 / 259(13.5)$ & 0.09 \\
\hline RF IgM & $88 / 364$ (24.2) & $14 / 283(4.9)$ & $<0.0001$ & $32 / 83(38.6)$ & $56 / 281(19.9)$ & 0.0005 \\
\hline RF IgA & $109 / 341(32.0)$ & $12 / 282(12.4)$ & $<0.0001$ & $34 / 74(45.9)$ & $75 / 267(28.0)$ & 0.004 \\
\hline \multicolumn{7}{|c|}{ Other laboratory analyses, mean \pm SD or $\mathrm{n}(\%)$} \\
\hline $\operatorname{IgA}, \mathrm{g} / 1$ & $2.90 \pm 1.46$ & $2.30 \pm 1.06$ & $<0.0001$ & $3.19 \pm 1.80$ & $2.82 \pm 1.32$ & 0.38 \\
\hline $\operatorname{IgG}, \mathrm{g} / 1$ & $13.63 \pm 5.54$ & $10.89 \pm 2.14$ & $<0.0001$ & $14.85 \pm 5.99$ & $13.25 \pm 5.35$ & 0.009 \\
\hline IgM, g/l & $1.24 \pm 1.26$ & $1.26 \pm 0.69$ & 0.0002 & $1.23 \pm 1.09$ & $1.24 \pm 1.31$ & 0.89 \\
\hline $\mathrm{C} 3, \mathrm{~g} / \mathrm{l}$ & $0.88 \pm 0.26$ & $1.06 \pm 0.21$ & $<0.0001$ & $0.90 \pm 0.26$ & $0.87 \pm 0.26$ & 0.33 \\
\hline $\mathrm{C} 4, \mathrm{~g} / \mathrm{l}$ & $0.15 \pm 0.08$ & $0.21 \pm 0.06$ & $<0.0001$ & $0.16 \pm 0.08$ & $0.15 \pm 0.07$ & 0.16 \\
\hline Lupus anticoagulant & $104(20.7)$ & ND & $<0.0001$ & $20(17.1)$ & $84(21.8)$ & 0.27 \\
\hline \multicolumn{7}{|l|}{ Cytokines, median (IQR) ${ }^{\#}$} \\
\hline $\mathrm{TNF}-\alpha, \mathrm{pg} / \mathrm{ml}$ & $4.5(3.1-6.2)$ & $2.3(2.0-2.8)$ & $<0.0001$ & $4.9(3.6-7.1)$ & $4.4(3.0-6.0)$ & 0.008 \\
\hline IL-6, pg/ml & $1.2(0.7-2.2)$ & $0.5(0.4-0.7)$ & $<0.0001$ & $1.5(0.8-3.0)$ & $1.1(0.6-2.0)$ & 0.009 \\
\hline $\mathrm{MCP}-4, \mathrm{pg} / \mathrm{ml}$ & $78(53.6-123.8)$ & $55.8(40.8-81.1)$ & $<0.0001$ & $94.9(66.9-131.3)$ & $74.7(52.4-120.0)$ & 0.019 \\
\hline MIP- $1 \beta, \mathrm{pg} / \mathrm{ml}$ & $72.7(50.8-108.1)$ & 43.7 (33.4-56.4) & $<0.0001$ & $81.1(54.8-123.6)$ & $68.9(50.3-105.1)$ & 0.020 \\
\hline IL-12/23p40, pg/ml & $180.6(122.9-286.3)$ & $131.0(99.5-179.3)$ & $<0.0001$ & $211.3(141.4-363.8)$ & $177.1(119.6-274.5)$ & 0.031 \\
\hline IP-10, pg/ml & $744.4(457.5-1497.4)$ & $352.13(258.9-478.9)$ & $<0.0001$ & $808.0(536.4-1911.7)$ & $726.4(440.8-1471.0)$ & 0.036 \\
\hline \multicolumn{7}{|c|}{ SLE manifestations (ever), $\mathrm{n}(\%)^{*}$} \\
\hline Malar rash & $246(48.8)$ & $0(0)$ & $<0.0001$ & $55(47.0)$ & $191(49.4)$ & 0.65 \\
\hline Discoid lesions & $88(17.5)$ & $0(0)$ & $<0.0001$ & $17(14.5)$ & $71(18.3)$ & 0.34 \\
\hline Photosensitivity & $318(63.1)$ & $57(17.8)$ & $<0.0001$ & $82(70)$ & $236(61)$ & 0.07 \\
\hline Oral ulcers & $168 / 502(33.5)$ & $11(3.4)$ & $<0.0001$ & $41(35)$ & $127 / 385(33)$ & 0.67 \\
\hline Arthritis & $406 / 503(80.7)$ & $13(4.0)$ & $<0.0001$ & $93(79.5)$ & $313 / 386(81.1)$ & 0.70 \\
\hline Pleuritis & $185 / 503(36.8)$ & $2(1)$ & $<0.0001$ & $50(42.7)$ & $135 / 386(35)$ & 0.12 \\
\hline Pericarditis & $88 / 503(17.5)$ & $0(0)$ & $<0.0001$ & $24(20.5)$ & $64 / 386(16.6)$ & 0.32 \\
\hline Nephritis & $202 / 503(40.2)$ & $1(0.3)$ & $<0.0001$ & $37 / 116(31.9)$ & $165(42.6)$ & 0.03 \\
\hline Psychosis & $10(2)$ & $2(1)$ & 0.11 & $1(1)$ & $9(2.3)$ & 0.46 \\
\hline Seizures & $49(9.7)$ & $5(1.6)$ & $<0.0001$ & $11(9.4)$ & $38(9.8)$ & 0.89 \\
\hline Leukopenia & $242(48)$ & $3(1)$ & $<0.0001$ & $67(57.3)$ & $175(45.2)$ & 0.02 \\
\hline Lymphopenia & $273(54.2)$ & $2(1)$ & $<0.0001$ & $68(58.1)$ & $205(53)$ & 0.32 \\
\hline Thrombocytopenia & $100(19.8)$ & $2(1)$ & $<0.0001$ & $24(20.5)$ & $76(19.6)$ & 0.83 \\
\hline Raynaud phenomenon & 195 (38.7) & $10(3.1)$ & $<0.0001$ & $51(43.6)$ & $144(37.2)$ & 0.21 \\
\hline Vasculitis & $57 / 498(11.4)$ & $0(0)$ & $<0.0001$ & $9 / 116(7.8)$ & $48 / 382(12.6)$ & 0.15 \\
\hline Interstitial lung disease & $24(4.8)$ & $0(0)$ & $<0.0001$ & $7(6.0)$ & $17(4.4)$ & 0.47 \\
\hline Peripheral neuropathy & $47 / 501(9.4)$ & $6(1.9)$ & $<0.0001$ & $18(15.4)$ & $29 / 384(7.5)$ & 0.01 \\
\hline \multicolumn{7}{|c|}{ Disease activity and damage indices } \\
\hline SLICC/ACR DI > 1 & $314 / 499(62.9)$ & ND & ND & $84 / 115(73.0)$ & $230 / 384(59.9)$ & 0.01 \\
\hline SLAM $>6$ & $298 / 501(59.5)$ & ND & ND & $78 / 116(67.2)$ & $220 / 385(57.1)$ & 0.05 \\
\hline SLEDAI $>2$ & $356 / 500(71.2)$ & ND & ND & $82 / 116(70.7)$ & 274/384 (71.4) & 0.88 \\
\hline
\end{tabular}

When measurements are missing, nos. are given as ratios (no. positive/total no. tested or available). Primary SS: fulfills criteria for primary Sjögren syndrome with the exception that patients are diagnosed with SLE. \# Cytokines were determined in 432 patients with SLE and 315 controls. * SLE criteria were determined according to ACR 1982 classification criteria. Values in bold face are statistically significant. SLE: systemic lupus erythematosus; SLE-sSS: SLE patients with secondary Sjögren syndrome; SLE-nonsSS: SLE patients without secondary Sjögren syndrome; aCL: anticardiolipin; Ig: immunoglobulin; $\beta_{2}$-GPI: $\beta_{2}$-glycoprotein I; C: complement factor; RF: rheumatoid factor; IQR: interquartile range; TNF: tumor necrosis factor; IL: interleukin; MCP: monocyte chemoattractant protein; MIP: macrophage inflammatory protein; IP: interferon $\gamma$-induced protein; SLICC/ACR DI: Systemic Lupus International Collaborating Clinics/American College of Rheumatology Damage Index; SLAM: Systemic Lupus Activity Measure; SLEDAI: SLE Disease Activity Index, ND: not determined. 


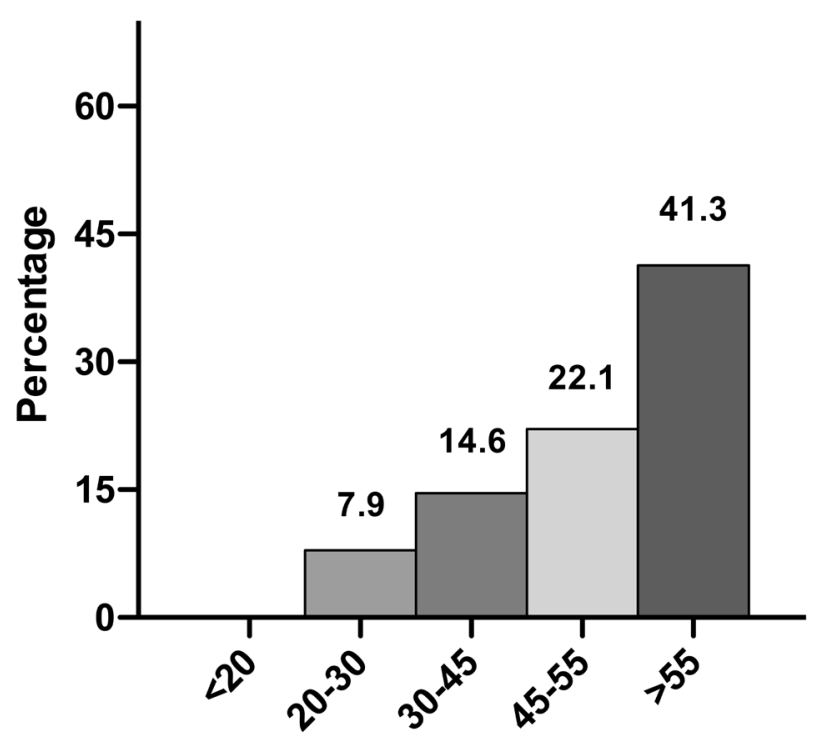

Age span, years

Figure 1. Age-stratified frequencies (\%) of SLE-sSS. SLE: systemic lupus erythematosus; SLE-sSS: SLE patients with secondary Sjögren syndrome.

Oral sicca symptoms. In the SSA/Ro52-, SSA/Ro60-, and SSB/La-positive groups, $49.2 \%, 52.7 \%$, and $50.9 \%$, respectively, presented with subjective oral sicca symptoms. Figures were similar for the triple SSA/SSB-positive patients (53.9\%; Supplementary Figure 1B, available with the online version of this article).

Sicca symptoms in triple SSA/SSB-negative SLE patients and controls. Among patients who were negative for all 3 SSA/SSB antibodies, $36.9 \%$ reported subjective oral sicca symptoms, $24.6 \%$ reported ocular sicca symptoms and $16.8 \%$ were diagnosed with sSS. In the control group, $7.5 \%$ reported oral and $7.1 \%$ ocular symptoms (Supplementary Figures 1A-B, available with the online version of this article).

\section{DISCUSSION}

According to the revised AECC ${ }^{15}$, SLE-sSS occurred in 23\% of patients in this large and well-defined cohort of consecutive SLE patients. It is a novel observation that, despite less internal organ involvement, higher levels of proinflammatory cytokines were present in the SLE-sSS group than in the SLE-nonsSS. We also confirm that older age, female sex, and SSA/SSB antibodies, not included in the AECC definition of sSS, were positively associated with SLE-sSS. However, notably a large minority (39\%) of SLE-sSS patients were negative for all $3 \mathrm{SSA} / \mathrm{SSB}$ antibodies.

To our knowledge, this is the first study to investigate whether systemic inflammation, as measured by cytokine levels, differs between SLE-sSS and SLE-nonsSS. Although SLE-sSS is often considered a less severe form of SLE, the levels of several proinflammatory cytokines - TNF- $\alpha$, IL-6, MCP-4, MIP-1 $\beta$, IL-12/IL23p40, and IP-10 - were higher in SLE-sSS than in SLE-nonsSS patients. As previously reported, the investigated cytokines were also upregulated in SLE versus controls, and they were positively associated with SLE disease activity ${ }^{4}$. TNF- and IL-6 have also been reported as high in $\mathrm{pSS}^{27}$. Interestingly, an IP-10 antagonist ameliorated the progression of autoimmune sialoadenitis in MRL/lpr mice ${ }^{28}$. Further, hypergammaglobulinemia, a well-known feature of $\mathrm{SS}^{29}$, was in our study consequently more common among SLE-sSS patients, although the levels of $\mathrm{IgG}$ were usually below $20 \mathrm{mg} / \mathrm{ml}$. We previously reported that low total IgM levels were associated with an SSA/SSB-positive profile in $\mathrm{SLE}^{29}$, but using the AECC, IgM levels were similar in SLE-sSS and SLE-nonsSS subgroups.

The occurrence of SLE-sSS in our study (23\%) is higher than that reported by most previous studies $(6-14 \%)^{18,20,30,31,32}$. An important reason for this discrepancy is likely attributable to our meticulous investigation procedures. In contrast to most studies, we measured tear and saliva production in all patients with SLE, regardless of whether subjective symptoms were present. It was a general finding that objectively reduced production of tear and saliva is more common than subjectively reported sicca symptoms. The high frequency of SLE-sSS is likely also affected by ethnicity. Our study population is mostly of European white origin and Baer, et al previously reported that in whites, as compared to other American ethnicities, frequencies of SLE-sSS were higher at $18 \%$ versus $14 \%{ }^{18}$.

The frequencies of SLE-sSS increased with age. Patients with SLE-sSS were on average 9 years older than SLE-nonsSS, both at disease onset and at inclusion. Similar age differences were also reported previously $18,20,30,33,34$. The difference in age at SLE onset did however not remain after controlling for present age. Comparable findings were reported by Baer, et al, who investigated a large multiethnic SLE $\operatorname{cohort}^{18}$ and by Manoussakis, et al ${ }^{30}$. In line with these observations, Nossent and Swaak showed a rising percentage of SLE-sSS during a 4-year followup of patients with SLE ${ }^{19}$. To further clarify whether sicca symptoms correlated with age, we stratified SLE-sSS, SLE-nonsSS, and controls by age. The results demonstrated that the prevalence of both subjective and objective sicca symptoms increase with age also in the SLE-nonsSS group and in controls. Thus, in some of the older patients with SLE-sSS, the AECC criteria may diagnose patients with sSS who have more age-related than immunological aberrations. Taken together, the present and previous studies demonstrate that SSS is an age-related complication among patients with SLE ${ }^{18,19}$.

The presence of SSA/SSB autoantibodies and their positive association to sicca symptoms is well recognized.Earlier studies reported that these antibodies occur together ${ }^{12,16}$. We observed that the prevalence of oral and ocular dryness was

Personal non-commercial use only. The Journal of Rheumatology Copyright $\subset$ 2020. All rights reserved. 
SSA/Ro-52 positivity in SLE-sSS and SLE-nonsSS

A

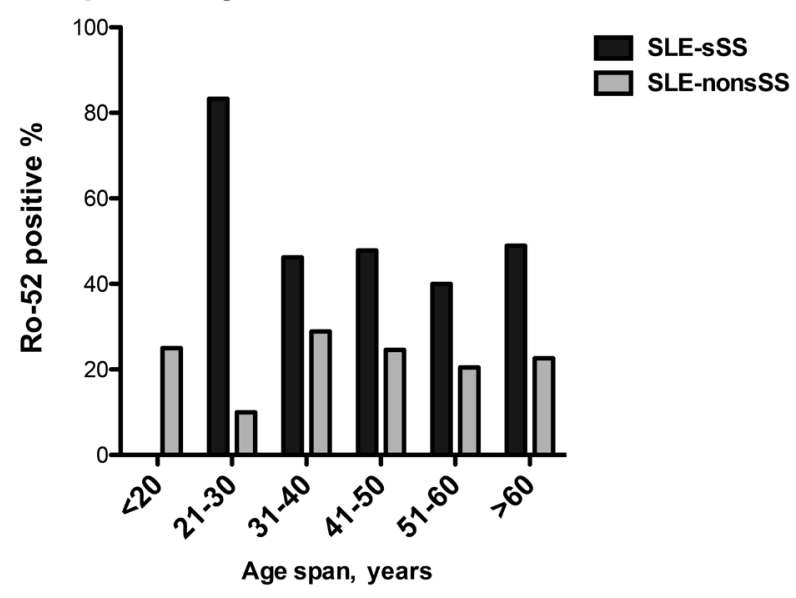

SSA/Ro-60 positivity in SLE-sSS and SLE-nonsSS

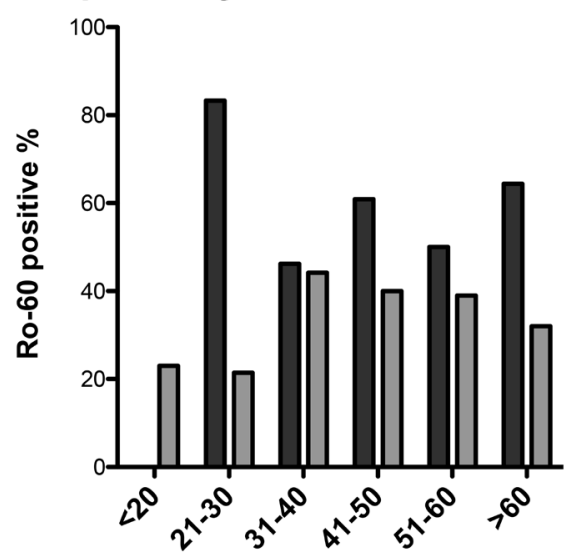

Age span, years

SLE-sSS

B

\section{SSB positivity in SLE-sSS and SLE-nonsSS}

C

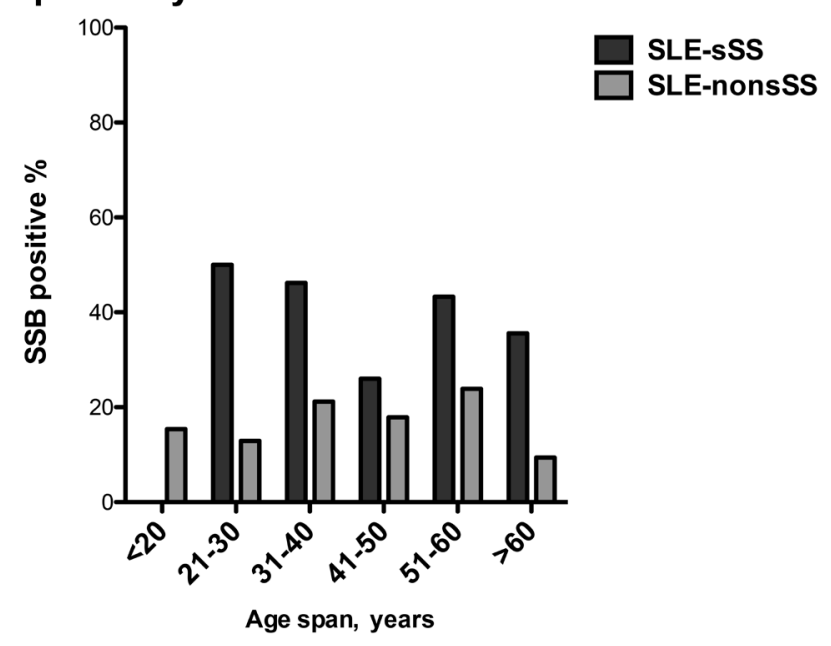

Figure 2. Occurrence of Sjögren-associated autoantibodies SSA/SSB in SLE-sSS and SLE-nonsSS patients, stratified for age. A. SSA-Ro52 positivity. B. SSA-Ro60 positivity. C. SSA-Ro52 positivity. SLE: systemic lupus erythematosus; SLE-sSS: SLE patients with secondary Sjögren syndrome; SLE-nonsSS: SLE patients without secondary Sjögren syndrome. 
Table 2. Frequency of organ manifestations in all SLE patients versus in SSA/SSB-positive patients with SLE.

\begin{tabular}{|c|c|c|c|c|c|c|c|c|}
\hline $\begin{array}{l}\text { SLE } \\
\text { Manifestations }\end{array}$ & $\begin{array}{l}\text { Missing } \\
\text { Data, n }\end{array}$ & $\begin{array}{l}\text { SLE Cohort, } \\
\mathrm{N}_{\text {total }}=504 \\
(100 \%)\end{array}$ & $\begin{array}{c}\text { anti-Ro52+ } \\
\mathrm{N}_{\text {Ro52+ }}=140 \\
(27.9 \%)\end{array}$ & $\mathrm{p}$ & $\begin{array}{c}\text { anti-Ro60+ } \\
\mathrm{N}_{\text {Ro60 }}=206 \\
(41.3 \%)\end{array}$ & $\mathrm{p}$ & $\begin{array}{c}\text { anti-La/SSB+ } \\
\mathrm{N}_{\mathrm{La} / \mathrm{SSB}}=113 \\
(22.6 \%)\end{array}$ & $\mathrm{p}$ \\
\hline Secondary SS & 0 & $117(23.2)$ & $56(47.9)$ & $<0.0001$ & $69 / 117(59.0)$ & $<0.0001$ & $44(37.6)$ & $<0.0001$ \\
\hline Malar rash & 0 & $246(48.8)$ & $58(41.4)$ & 0.04 & $103(50.0)$ & 0.67 & $54(47.8)$ & 0.78 \\
\hline Discoid lesions & 0 & $88(17.5)$ & $27(19.3)$ & 0.52 & $46(22.3)$ & 0.02 & $25(22.1)$ & 0.14 \\
\hline Photosensitivity & 0 & $318(63.1)$ & $94(67.1)$ & 0.26 & $142 / 201(68.9)$ & 0.02 & 78/103 (69.0) & 0.14 \\
\hline Oral ulcers & 2 & $168(33.5)$ & $50(35.7)$ & 0.46 & 68/201 (33.0) & 0.87 & $40 / 103(35.4)$ & 0.58 \\
\hline Arthritis & 1 & $406(80.7)$ & $109(77.9)$ & 0.33 & $161 / 201(78.2)$ & 0.26 & $80 / 103(70.8)$ & 0.002 \\
\hline Pleuritis & 1 & $185(36.8)$ & $53(37.9)$ & 0.75 & $73 / 201(35.4)$ & 0.61 & $48 / 103(42.5)$ & 0.14 \\
\hline Pericarditis & 1 & $88(17.5)$ & $27(19.3)$ & 0.48 & 33/201 (16.0) & 0.47 & 18/103 (15.9) & 0.63 \\
\hline Nephritis & 1 & $202(40.2)$ & $47 / 139(33.8)$ & 0.07 & $72 / 205(35.1)$ & 0.05 & 29/112 (25.9) & 0.0005 \\
\hline Psychosis & 0 & $10(2)$ & $2(1.4)$ & 0.57 & $5(2.4)$ & 0.57 & $1(0.9)$ & 0.33 \\
\hline Seizures & 0 & $49(9.7)$ & $7(5.0)$ & 0.02 & $15(7.3)$ & 0.11 & $7(6.2)$ & 0.14 \\
\hline Leukopenia & 0 & $242(48.0)$ & $77(55.0)$ & 0.04 & $117(56.8)$ & 0.0006 & $71(62.8)$ & 0.0003 \\
\hline Lymphopenia & 0 & $273(54.2)$ & $85(60.7)$ & 0.05 & $121(58.7)$ & 0.06 & $70(61.9)$ & 0.05 \\
\hline Thrombocytopenia & 0 & $100(19.8)$ & $25(17.9)$ & 0.46 & $42(20.4)$ & 0.79 & $23(20.4)$ & 0.90 \\
\hline $\mathrm{RP}$ & 0 & $195(38.7)$ & $55(39.3)$ & 0.87 & $82(39.8)$ & 0.60 & $41(36.3)$ & 0.57 \\
\hline Vasculitis* & 6 & $57(11.4)$ & $18 / 138(13.0)$ & 0.50 & $31 / 203(15.3)$ & 0.03 & $16 / 112(14.3)$ & 0.29 \\
\hline Interstitial lung disease & 0 & $24(4.8)$ & $10(7.1)$ & 0.12 & $10(4.9)$ & 0.96 & $6(5.3)$ & 0.76 \\
\hline Peripheral neuropathy & 3 & $47(9.4)$ & $15 / 139(10.8)$ & 0.45 & $26 / 204(12.7)$ & 0.02 & 13/112 (11.6) & 0.32 \\
\hline
\end{tabular}

Numbers are given as ratios (no. positive/total no. tested) when measurements are missing. Values are n (\%) unless otherwise specified. Values in bold face are statistically significant. * All clinical vasculitis. SLE: systemic lupus erythematosus; SS: Sjögren syndrome; RP: Raynaud phenomenon; ILD: interstitial lung disease.

more pronounced in patients who are positive for all 3 antibodies (SSA/Ro52, SSA/Ro60, and SSB/La). Our study thus supports the presence of an autoantibody cluster related to the SLE-sSS subset ${ }^{10,11,29}$. Although the presence of the SSA/SSB antibodies was associated with sicca symptoms, a considerable fraction of patients with SLE-sSS (39\%) were negative for all $3 \mathrm{SSA} / \mathrm{SSB}$ antibodies. We also report higher frequencies of IgM and IgA RF in patients with SLE-sSS. Thus, the presence of sicca symptoms is not exclusively related to SSA/SSB antibodies, but also to increasing age, $\mathrm{RF}$, and possibly to other unidentified factors.

Overall organ damage was more severe in the SLE-sSS group, but this difference did not remain after age adjustment. Regarding disease activity, we performed 2 validated indices: $\mathrm{SLEDAI}^{23}$, a qualitative (presence vs absence) recording of symptoms and laboratory aberrations; and $\mathrm{SLAM}^{22}$, which grades symptom severity and includes subjective symptoms such as fatigue, headache, and arthralgia. SLEDAI measurements did not differ, whereas with SLAM, higher disease activity scores were observed in the SLE-sSS than in the SLE-nonsSS subgroup. This observation could possibly be explained by the inflammatory state, which may cause subjective and general symptoms such as muscle and joint pain, headache and fatigue, which are included in the SLAM but not in the SLEDAI index.

Consistent with earlier studies ${ }^{18,30,34}$, the female predominance was more pronounced among SLE-sSS patients as compared to SLE-nonsSS (96\% vs $84 \%$ ). The very high percentage of female patients with SLE-sSS (96\%) is similar to reports in $\mathrm{pSS}^{30}$. The average age of disease onset for SLE-sSS was older than for SLE-nonsSS $(40.4 \pm 15.6$ vs $33.9 \pm 14.9$ yrs) but still considerably younger than the average age of onset for pSS, which is 55 years ${ }^{35,36}$.

In clinical practice, it is often difficult to delineate pSS from SLE-sSS. Organ manifestations commonly reported in pSS are fever, lymphadenopathy, parotid gland enlargement, Raynaud phenomenon, interstitial lung disease, peripheral neuropathy, and vasculitis ${ }^{37,38,39}$. All these clinical features, except parotid gland enlargement, were investigated in our present study, but only peripheral neuropathy differed and was more frequent in SLE-sSS than in SLE-nonsSS, demonstrating that the majority of these manifestations are shared between SLE and pSS. Of SLE manifestations, leukopenia was more common in the SLE-sSS group and there was also a positive trend for photosensitivity, both of which have been associated with the type I interferon signature. Nephritis was, in accordance with previous studies, less frequent in the SLE-sSS group, and anti-dsDNA positivity showed a similar trend ${ }^{18,20,30,34}$. We conclude that patients with SLE-sSS and patients with pSS have many similarities and it is not surprising that among the patients with SLE-sSS, according to AECC, $21 \%$ also fulfilled the criteria for pSS, if we disregard the fact that they are diagnosed with SLE (a prerequisite for being evaluated for $\mathrm{pSS}$ ).

The strengths of this study are the objective measurements of sicca symptoms in all patients with SLE, according to the AECC criteria and recommended practice in Sweden, in a well-characterized consecutively collected cohort of patients with SLE. However, if the investigating rheumatologist did not consider sSS to be present, we did not refer

Personal non-commercial use only. The Journal of Rheumatology Copyright $(\subset) 2020$. All rights reserved. 


\section{Ocular symptoms in Controls, SLE-nonsSS and SLE-sSS}

A

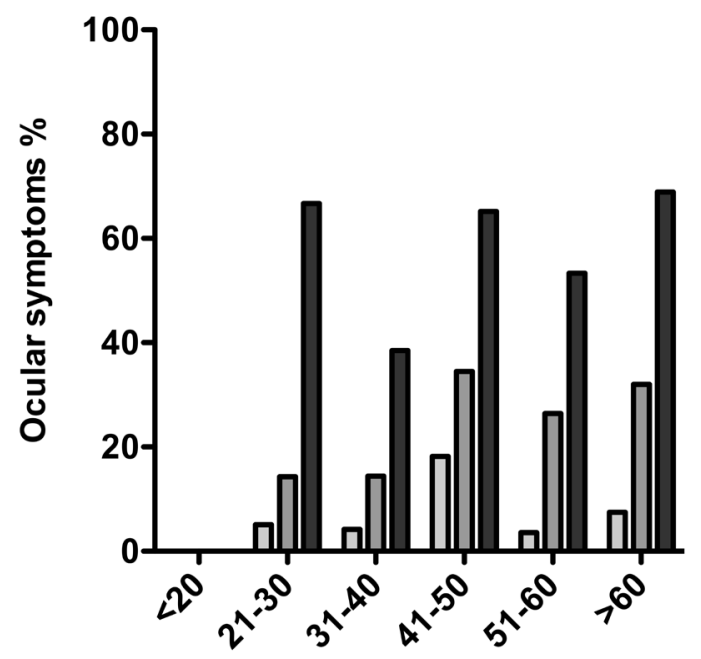

$\square$ Controls

$\square$ SLE-nonsSS

SLE-sSS

Age span, years

\section{Positive Schirmer's test in Controls, SLE-nonsSS and SLE-sSS}

B

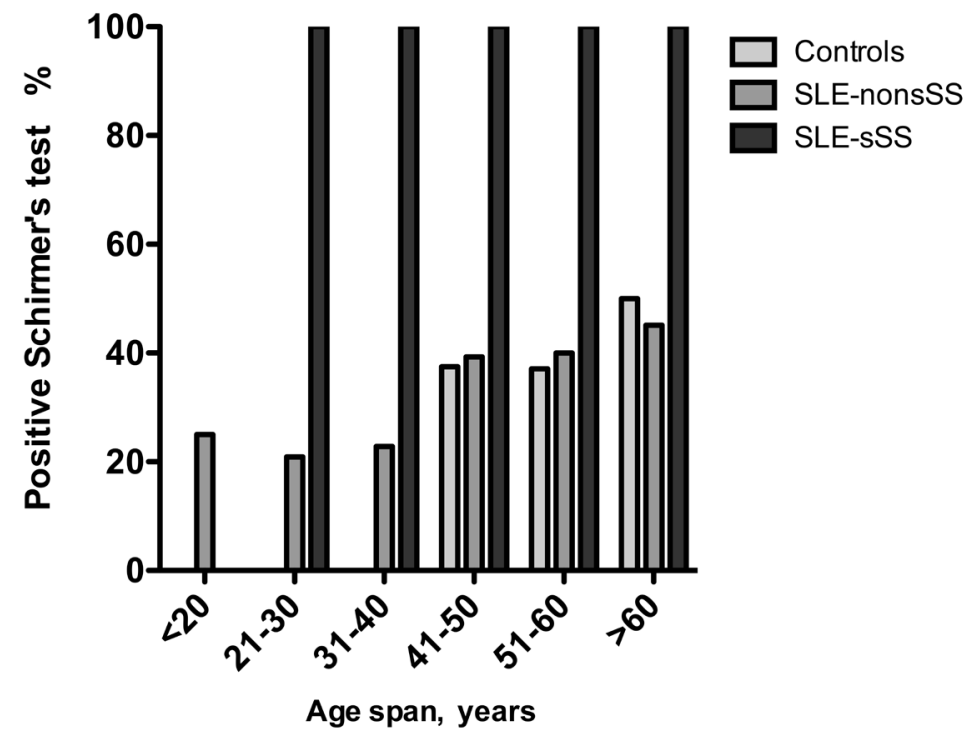

Figure 3. Subjective and objective ocular symptoms in patients with SLE-sSS and SLE-nonsSS, and in controls. A. Subjective ocular symptoms in controls, SLE-nonsSS, and SLE-sSS, as defined by a validated questionnaire according to item 1 in the American-European Consensus Criteria (AECC) ${ }^{15}$. B. Objective ocular symptoms in controls, SLE-nonsSS, and SLE-sSS, measured according to item 3 in AECC by Schirmer's test, where a positive value is given to an amount of $<5 \mathrm{~mm}$ of tears collected during $5 \mathrm{~min}$ from one or both eyes. SLE: systemic lupus erythematosus; SLE-sSS: SLE patients with secondary Sjögren syndrome; SLE-nonsSS: SLE patients without secondary Sjögren syndrome.

to an ophthalmologist for Rose Bengal staining or other ocular dye scores, to achieve full potential for all patients to fulfill item III (ocular signs) in the classification criteria. Similarly, salivary gland biopsies were only performed when the investigating rheumatologist suspected that sSS could be present, despite not fulfilling items III and V (salivary gland involvement) according to WUSF. We are aware that previous studies have reported a lower sensitivity if 
Oral symptoms in Controls, SLE-nonsSS and SLE-sSS

A

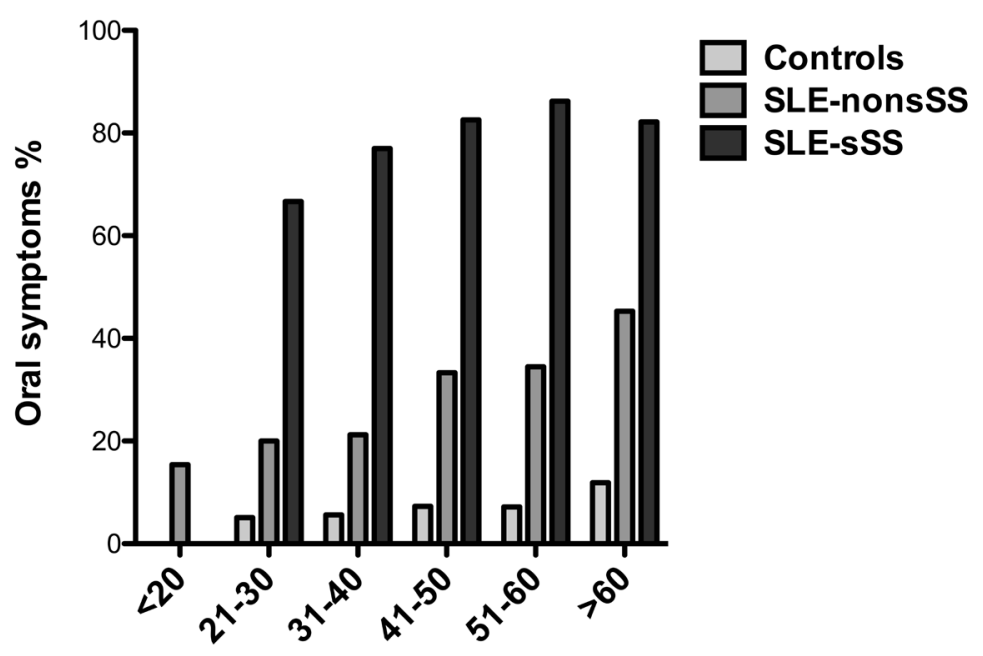

Age span, years

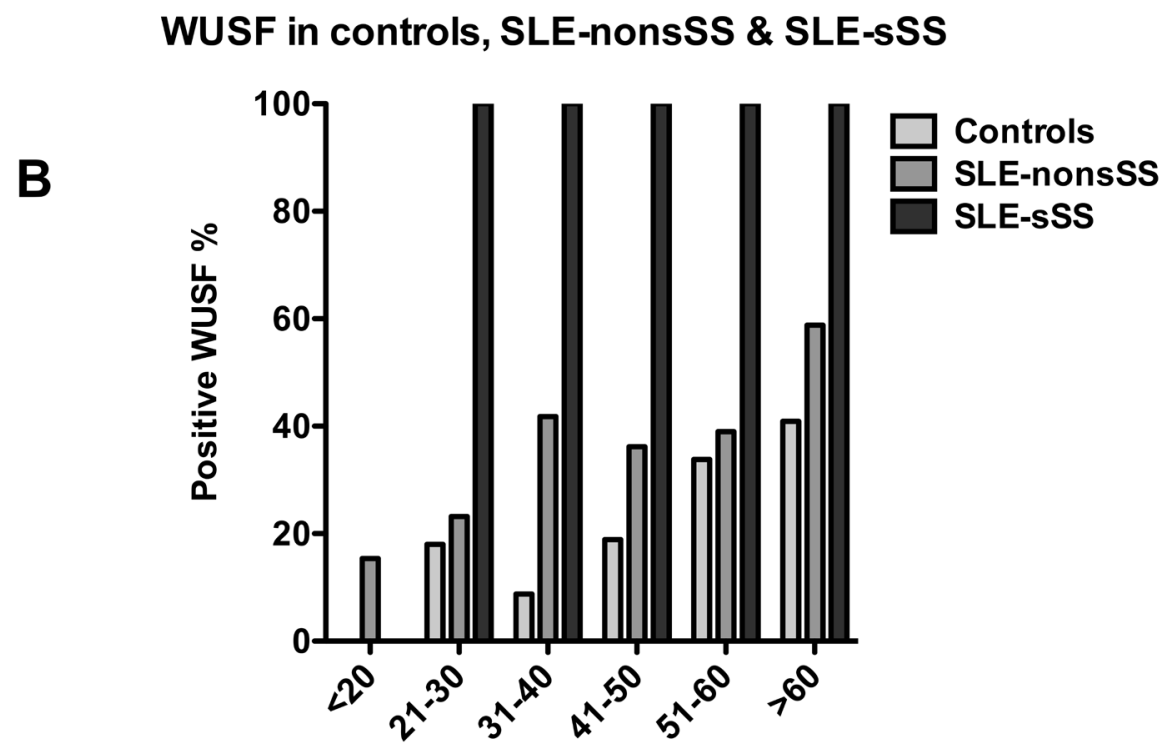

Age span, years

Figure 4. Subjective and objective oral symptoms in patients with SLE-sSS and SLE-nonsSS, and in controls. Subjective oral symptoms in controls, SLE-nonsSS, and SLE-sSS are as defined by a validated questionnaire according to item 2 in the American-European Consensus Criteria (AECC) ${ }^{15}$. Objective oral symptoms in controls, SLE-nonsSS, and SLE-sSS as measured according to item 5 in $\mathrm{AECC}^{15}$ by WUSF over 15 min, where a positive value is given to an amount of $<1.5 \mathrm{ml}$ of saliva collected during $15 \mathrm{~min}$. SLE: systemic lupus erythematosus; SLE-sSS: SLE patients with secondary Sjögren syndrome; SLE-nonsSS: SLE patients without secondary Sjögren syndrome; WUSF: whole unstimulated salivary flow.

only Schirmer's test and WUSF are used $^{40}$, and we have considered the risk for misclassification, but the rheumatologists assessed these patients clinically as SLE-nonsSS. To further illustrate this issue, we excluded the 132 patients in whom a biopsy could have made a difference, but this did not essentially change the characteristic differences between SLE-sSS and SLE-nonsSS. Excluding these patients would also have changed the study's cross-sectional and consecutive design. Finally, we did not measure saliva and tear production in controls without sicca symptom. Because 
positive objective measures were more common than subjective complaints, we may have underestimated the number of controls with impaired saliva/tear production.

Our investigations of the SLE-sSS subset demonstrate that it affects roughly one-quarter of patients with SLE, and the frequency increases with age. Autoantibodies SSA/SSB occurin themajority, butalarge minority (39\%)were SSA/SSB-negative. SLE-sSS shares many features with pSS such as a striking female predominance, older age at onset, and neuropathy. It is a novel observation, with possible therapeutic implications, that an inflammatory state occurs in SLE-sSS with higher levels of proinflammatory cytokines than in SLE-nonsSS.

\section{ACKNOWLEDGMENT}

We are grateful to Eva Jemseby for management of blood samples and to Jill Gustafsson, Sonia Möller, and Susanne Pettersson for coordination and blood sampling. We thank Johanna Gustafsson and Ola Börjesson for inclusion of patients.

\section{ONLINE SUPPLEMENT}

Supplementary material accompanies the online version of this article.

\section{REFERENCES}

1. Lisnevskaia L, Murphy G, Isenberg D. Systemic lupus erythematosus. Lancet 2014;384:1878-88.

2. Ronnblom L, Eloranta ML, Alm GV. The type I interferon system in systemic lupus erythematosus. Arthritis Rheum 2006;54:408-20.

3. Leffler J, Bengtsson AA, Blom AM. The complement system in systemic lupus erythematosus: an update. Ann Rheum Dis 2014;73:1601-6.

4. Idborg H, Eketjall S, Pettersson S, Gustafsson JT, Zickert A, Kvarnstrom M, et al. TNF- $\alpha$ and plasma albumin as biomarkers of disease activity in systemic lupus erythematosus. Lupus Sci Med 2018;5:e000260.

5. Grosso G, Vikerfors A, Woodhams B, Adam M, Bremme K, Holmstrom M, et al. Thrombin activatable fibrinolysis inhibitor (TAFI) - a possible link between coagulation and complement activation in the antiphospholipid syndrome (APS). Thromb Res 2017;158:168-73.

6. Bernatsky S, Boivin JF, Joseph L, Manzi S, Ginzler E, Gladman $\mathrm{DD}$, et al. Mortality in systemic lupus erythematosus. Arthritis Rheum 2006;54:2550-7.

7. Gustafsson JT, Simard JF, Gunnarsson I, Elvin K, Lundberg IE, Hansson LO, et al. Risk factors for cardiovascular mortality in patients with systemic lupus erythematosus, a prospective cohort study. Arthritis Res Ther 2012;14:R46.

8. Tektonidou MG, Wang Z, Ward MM. Brief report: trends in hospitalizations due to acute coronary syndromes and stroke in patients with systemic lupus erythematosus, 1996 to 2012. Arthritis Rheumatol 2016;68:2680-5.

9. Heaton JM. Sjogren's syndrome and systemic lupus erythematosus. Br Med J 1959;1:466-9.

10. Ching KH, Burbelo PD, Tipton C, Wei C, Petri M, Sanz I, et al. Two major autoantibody clusters in systemic lupus erythematosus. PLoS One 2012;7:e32001.

11. To CH, Petri M. Is antibody clustering predictive of clinical subsets and damage in systemic lupus erythematosus? Arthritis Rheum 2005;52:4003-10.

12. Artim-Esen B, Cene E, Sahinkaya Y, Ertan S, Pehlivan O, Kamali $\mathrm{S}$, et al. Cluster analysis of autoantibodies in 852 patients with systemic lupus erythematosus from a single center. J Rheumatol 2014;41:1304-10.
13. Idborg H, Zandian A, Sandberg AS, Nilsson B, Elvin K, Truedsson $\mathrm{L}$, et al. Two subgroups in systemic lupus erythematosus with features of antiphospholipid or Sjögren's syndrome differ in molecular signatures and treatment perspectives. Arthritis Res Ther 2019;21:62.

14. Li PH, Wong WH, Lee TL, Lau CS, Chan TM, Leung AM, et al. Relationship between autoantibody clustering and clinical subsets in SLE: cluster and association analyses in Hong Kong Chinese. Rheumatology 2013;52:337-45.

15. Vitali C, Bombardieri S, Jonsson R, Moutsopoulos HM, Alexander EL, Carsons SE, et al; European Study Group on Classification Criteria for Sjögren's Syndrome. Classification criteria for Sjögren's syndrome: a revised version of the European criteria proposed by the American-European consensus group. Ann Rheum Dis 2002;61:554-8.

16. Eriksson C, Kokkonen H, Johansson M, Hallmans G, Wadell G, Rantapaa-Dahlqvist S. Autoantibodies predate the onset of systemic lupus erythematosus in northern Sweden. Arthritis Res Ther 2011;13:R30.

17. Arbuckle MR, McClain MT, Rubertone MV, Scofield RH, Dennis GJ, James JA, et al. Development of autoantibodies before the clinical onset of systemic lupus erythematosus. N Engl J Med 2003;349:1526-33.

18. Baer AN, Maynard JW, Shaikh F, Magder LS, Petri M. Secondary Sjogren's syndrome in systemic lupus erythematosus defines a distinct disease subset. J Rheumatol 2010;37:1143-9.

19. Nossent JC, Swaak AJ. Systemic lupus erythematosus vii: Frequency and impact of secondary Sjøgren's syndrome. Lupus 1998;7:231-4.

20. Pan HF, Ye DQ, Wang Q, Li WX, Zhang N, Li XP, et al. Clinical and laboratory profiles of systemic lupus erythematosus associated with Sjögren syndrome in China: a study of 542 patients. Clin Rheumatol 2008;27:339-43.

21. Tan EM, Cohen AS, Fries JF, Masi AT, McShane DJ, Rothfield NF, et al. The 1982 revised criteria for the classification of systemic lupus erythematosus. Arthritis Rheum 1982;25:1271-7.

22. Liang MH, Socher SA, Roberts WN, Esdaile JM. Measurement of systemic lupus erythematosus activity in clinical research. Arthritis Rheum 1988;31:817-25.

23. Bombardier C, Gladman DD, Urowitz MB, Caron D, Chang CH. Derivation of the SLEDAI. A disease activity index for lupus patients. The Committee on Prognosis Studies in SLE. Arthritis Rheum 1992;35:630-40.

24. Gladman D, Ginzler E, Goldsmith C, Fortin P, Liang M, Urowitz $\mathrm{M}$, et al. The development and initial validation of the Systemic Lupus International Collaborating Clinics/American College of Rheumatology Damage Index for systemic lupus erythematosus. Arthritis Rheum 1996;39:363-9.

25. Skopouli FN, Siouna-Fatourou HI, Ziciadis C, Moutsopoulos HM Evaluation of unstimulated whole saliva flow rate and stimulated parotid flow as confirmatory tests for xerostomia. Clin Exp Rheumatol 1989;7:127-9.

26. Miyakis S, Lockshin MD, Atsumi T, Branch DW, Brey RL, Cervera $\mathrm{R}$, et al. International consensus statement on an update of the classification criteria for definite antiphospholipid syndrome (APS). J Thromb Haemost 2006;4:295-306.

27. Szodoray P, Alex P, Brun JG, Centola M, Jonsson R. Circulating cytokines in primary Sjögren's syndrome determined by a multiplex cytokine array system. Scand J Immunol 2004;59:592-9.

28. Muraoka M, Hasegawa H, Kohno M, Inoue A, Miyazaki T, Terada $\mathrm{M}$, et al. IK cytokine ameliorates the progression of lupus nephritis in MRL/lpr mice. Arthritis Rheum 2006;54:3591-600.

29. Gronwall C, Hardt U, Gustafsson JT, Elvin K, Jensen-Urstad K, Kvarnstrom M, et al. Depressed serum IgM levels in SLE are restricted to defined subgroups. Clin Immunol 2017;183:304-15. 
30. Manoussakis MN, Georgopoulou C, Zintzaras E, Spyropoulou M, Stavropoulou A, Skopouli FN, et al. Sjögren's syndrome associated with systemic lupus erythematosus: clinical and laboratory profiles and comparison with primary Sjögren's syndrome. Arthritis Rheum 2004;50:882-91

31. Gilboe IM, Kvien TK, Uhlig T, Husby G. Sicca symptoms and secondary Sjögren's syndrome in systemic lupus erythematosus: comparison with rheumatoid arthritis and correlation with disease variables. Ann Rheum Dis 2001;60:1103-9.

32. Alani H, Henty JR, Thompson NL, Jury E, Ciurtin C. Systematic review and meta-analysis of the epidemiology of polyautoimmunity in Sjögren's syndrome (secondary Sjögren's syndrome) focusing on autoimmune rheumatic diseases. Scand J Rheumatol 2018; 47:141-54.

33. Szanto A, Szodoray P, Kiss E, Kapitany A, Szegedi G, Zeher M. Clinical, serologic, and genetic profiles of patients with associated Sjögren's syndrome and systemic lupus erythematosus. Hum Immunol 2006;67:924-30.

34. Xu D, Tian X, Zhang W, Zhang X, Liu B, Zhang F. Sjogren's syndrome-onset lupus patients have distinctive clinical manifestations and benign prognosis: a case-control study. Lupus 2010;19:197-200.

35. Qin B, Wang J, Yang Z, Yang M, Ma N, Huang F, et al.
Epidemiology of primary Sjögren's syndrome: a systematic review and meta-analysis. Ann Rheum Dis 2015;74:1983-9.

36. Kvarnstrom M, Ottosson V, Nordmark B, Wahren-Herlenius M. Incident cases of primary Sjögren's syndrome during a 5-year period in Stockholm County: a descriptive study of the patients and their characteristics. Scand J Rheumatol 2015;44:135-42.

37. Fox RI. Sjögren's syndrome. Lancet 2005;366:321-31.

38. Ramos-Casals M, Brito-Zeron P, Seror R, Bootsma H, Bowman SJ, Dorner T, et al; EULAR Sjögren Syndrome Task Force. Characterization of systemic disease in primary Sjögren's syndrome: EULAR-SS task force recommendations for articular, cutaneous, pulmonary and renal involvements. Rheumatology 2015;54:2230-8.

39. Maldini C, Seror R, Fain O, Dhote R, Amoura Z, De Bandt M, et al. Epidemiology of primary Sjogren's syndrome in a French multiracial/multiethnic area. Arthritis Care Res 2014;66:454-63.

40. Shiboski SC, Shiboski CH, Criswell L, Baer A, Challacombe S, Lanfranchi H, et al; Sjögren's International Collaborative Clinical Alliance (SICCA) Research Groups. American College of Rheumatology classification criteria for Sjögren's syndrome: a data-driven, expert consensus approach in the Sjögren's international collaborative clinical alliance cohort. Arthritis Care Res 2012;64:475-87. 